\title{
Intention Classification of a User of a Walking Assist Cart by using Support Vector Machine
}

\author{
Noritaka Sato*, Tomoki Yokotani, Yoshifumi Morita \\ Department of Electrical and Mechanical Engineering, Nagoya Institute of Technology, Gokiso-cho, Syowa-ku, Nagoya, Aichi 466-8555, Japan
}

\section{ARTICLE INFO}

\section{Article History}

Received 10 November 2019

Accepted 20 February 2020

\section{Keywords}

Walking assist cart support vector machine intention classification artificial intelligence

\begin{abstract}
To develop better assist function for a walking assist cart, we focused on the prediction of the intention of a user. As the first step of the research, the forces and torques to the cart from the user's hands, and the rotational velocities of the wheels are sensing. And the support vector machine is used for intention classification. As a result, we confirmed that our method was able to predict the intention of the user with enough accuracy.
\end{abstract}

(C) 2020 The Authors. Published by Atlantis Press SARL. This is an open access article distributed under the CC BY-NC 4.0 license (http://creativecommons.org/licenses/by-nc/4.0/).

\section{INTRODUCTION}

With the improvement of medical technology and living environment, the ratio of elderly people is increasing [1]. Elderly people have weak legs, so they take less walks and shopping, and tend to stay home. This makes walking more difficult. Moreover, the number of elderly people living alone is increasing, and many elderly people cannot get help from others. It is essential for the elderly to be able to live independently and comfortably.

One way to solve this problem is to use a walking assist cart. To improve the walking assist cart, some researchers and some companies add an electric assist function to the cart $[2,3]$. This research started as a joint research with SHINTEC HOZUMI Co., Ltd. (Miyoshi, Aichi, Japan). Figure 1 shows the current walking assist cart of SHINTEC HOZUMI Co., Ltd. [4]. This cart can perform assist control such as deceleration on downhill.

However, there is a problem in the current cart that a sense of incongruity arises because the assist according to the intention of the user cannot be performed. In this research, the final goal is to develop an algorithm that estimates human intention based on sensor information on a cart and enables an assistant to feel comfortable.

In this paper, as the first step of the research, we aim to predict the state of the cart after $200 \mathrm{~ms}$ using the wheel speed and the force on the handles of the cart. Specifically, we use a Support Vector Machine (SVM) to create a learning model from data collected in advance and make predictions. We intend to achieve the final goal by increasing the number of motions that can be predicted. Note that the $200 \mathrm{~ms}$ were determined based on discussions with the joint research partner.

\section{DATA COLLECTION}

In this study, we performed data collection experiments using real machines for learning SVM. Figure 2 shows the walking vehicle used in this study. Although this cart has an assist function, the assist function was not used in the data collection experiment.

The motors on the left and right wheels of the cart are equipped with encoders. In this study, the inexpensive force sensor PFS055YA251U6 made by Leptrino was attached to the left and right handles as shown in Figure 3.

The subject moved the cart forward, backward, and turn as shown in Figures 4 and 5, and we recorded the forces and torques on the left and right handles, and the left and right wheel speeds. The turning radius was $0.5 \mathrm{~m}$. Five subjects performed each operation three times.

Figure 6 shows an example of the acquired data. When the cart goes straight from the stop state (between 0.2 and $0.3 \mathrm{~s}$ ), the value of the force sensor changes before the cart starts moving. Therefore, we think that the state of the cart can be estimated by using the information of the current wheel speed and the force sensor.

\section{PROPOSED METHOD}

In this study, the state of the cart $200 \mathrm{~ms}$ ahead (stop, forward, reverse, right turn, or left turn) will be estimated using SVM. Therefore, the state of the walking car at each time was determined from the acquired data and the video taken during the data collection experiment. After that, the state $200 \mathrm{~ms}$ ahead of each time was determined as the correct state for estimation by using SVM.

Figure 7 shows a scatter diagram of the force in the front-rear direction on the left and right handles after the noise is removed 


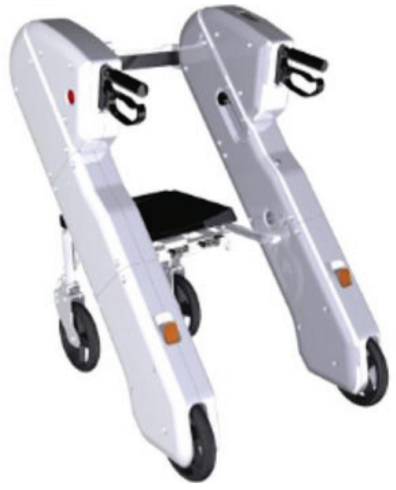

Figure 1 Walking assist cart of SHINTEC HOZUMI Co., Ltd.

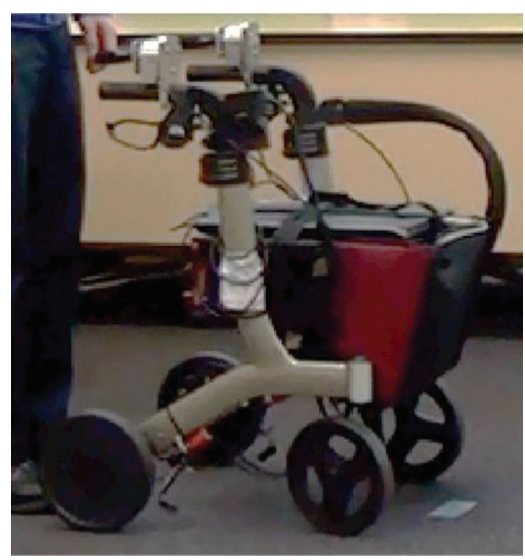

Figure 2 Assist cart used in the data collection experiment.
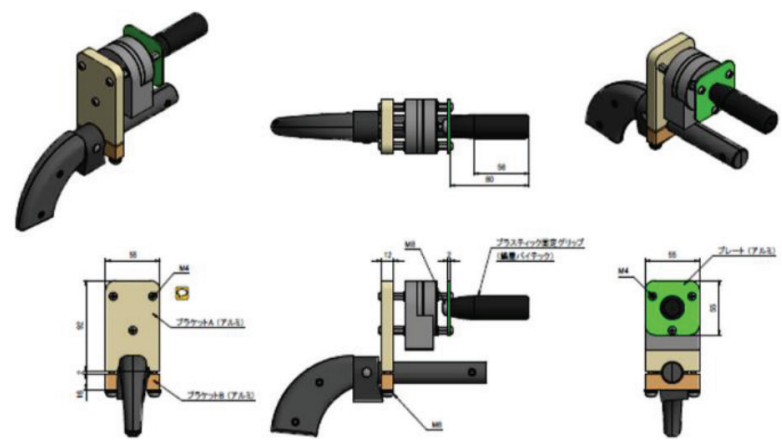

Figure 3 Attaching method of the force sensor.

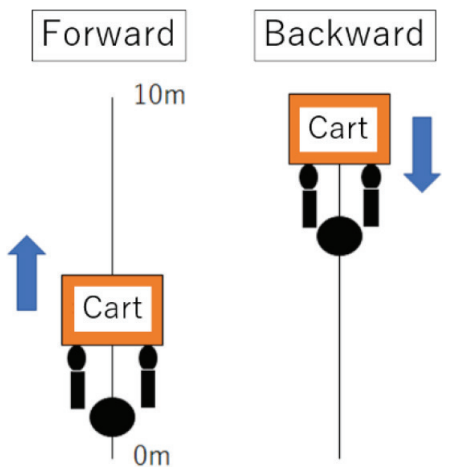

Figure 4 Forward and backward motion in the data collection experiment.

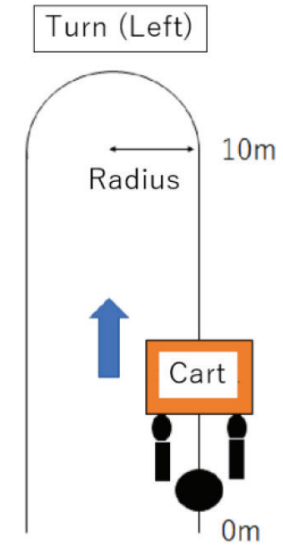

Figure 5 Turn motion in the data collection experiment.

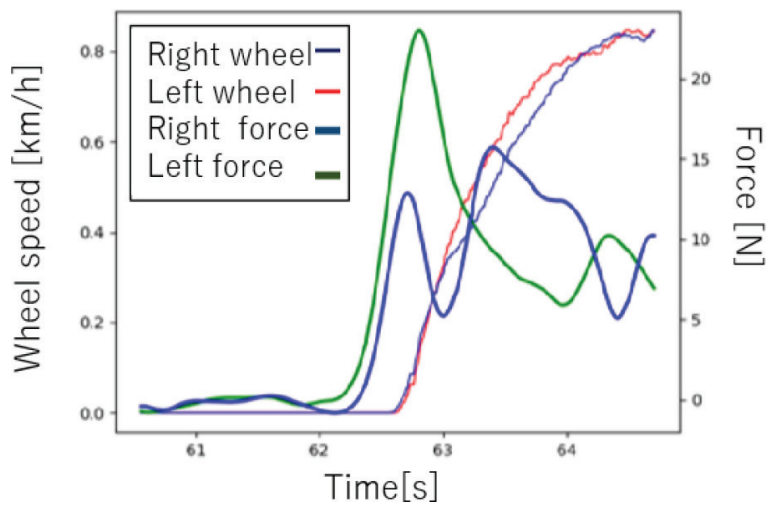

Figure 6 Example of acquired data.

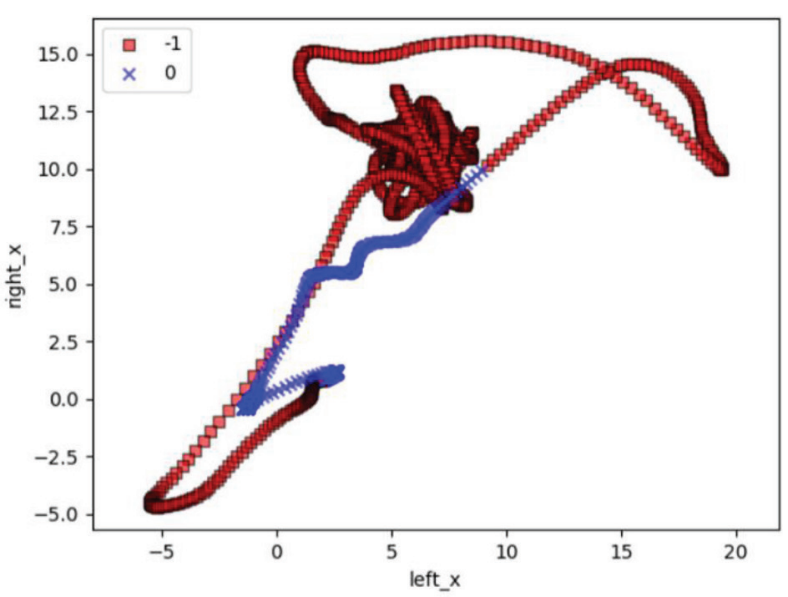

Figure 7 Scatter diagram of the force in the front-rear direction on the left and right handles.

by the low-pass filter. Here, 0 and -1 mean stop and go straight after $200 \mathrm{~ms}$, respectively. It was determined that the point in a certain state was within a certain range, and that it could be estimated $200 \mathrm{~ms}$ ahead by clustering with SVM. In Figure 7, a graph was drawn using two representative values, but in actuality, estimation by using SVM was performed using 14 data, including the values of the left and right 6-axis force sensors and the left and right wheel speeds, as inputs. 


\begin{tabular}{|c|c|c|c|c|c|}
\hline & & Estimat & d valu & & \\
\hline & -1 & 0 & 1 & 2 & 3 \\
\hline-1 & 80323 & 771 & 72 & 34 & 0 \\
\hline 0 & 284 & 71919 & 0 & 77 & 130 \\
\hline 1 & 407 & 0 & 4453 & 0 & 0 \\
\hline 2 & 827 & 175 & 0 & 9290 & 59 \\
\hline 3 & 0 & 180 & 0 & 0 & 20544 \\
\hline
\end{tabular}

Figure 8 Confusion matrix classified by SVM.

"scikit-learn", which is the Python library is used for the calculation of the SVM in this study.

\section{RESULTS}

Cross-validation was performed on the data divided into 15 pieces. Figure 8 shows a confusion matrix classified by SVM. The numbers on the label are -1 for forward, 0 for stop, 1 for left turn, 2 for right turn, and 3 for backward. The numbers in the matrix represent how many points were output as which labels.

Using this value, the correct answer rate for each cart condition is calculated as $98.9 \%$ for forward, $99.3 \%$ for stop, $91.6 \%$ for left turn, $89.7 \%$ for right turn, and $99.1 \%$ for backward. The overall correct answer rate is $98.4 \%$. From the above, it is understood that the state of the cart $200 \mathrm{~ms}$ ahead can be accurately predicted by the proposed method.

\section{CONCLUSION}

The final goal of this study was to develop an algorithm that predicts human intentions and assists a cart that makes people feel

\section{AUTHORS INTRODUCTION}

\section{Dr. Noritaka Sato}

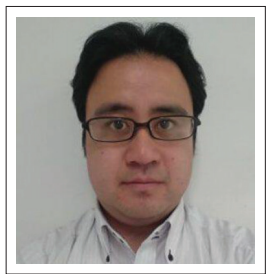

He received Doctor of Engineering degree from The University of ElectroCommunications in 2009. He was a program-specific Assistant Professor of Kyoto University in 2009-2011, and is an assistant professor of Nagoya Institute of Technology since 2011. His research interests include human robot interaction for rescue robots, rehabilitation-assist robots and industrial robots. $\mathrm{He}$ is a member of the IEEE, RSJ and SICE.

\section{Mr. Tomoki Yokotani}

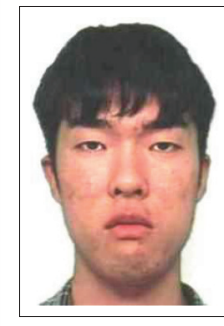

He received Master of Engineering degree from Nagoya Institute of Technology in 2019. His research interests include autonomous control system of rescue robots and human robot interaction of robotic system. more comfortable. The movements were classified by SVM based on wheel speed and force/torque on the handle. We created an SVM program using Python library named scikit-learn and applied it to the collected data. The results showed that the correct answer rate of $98.4 \%$ was obtained as a whole, and that the state of the cart could be accurately estimated.

In the future, the prediction accuracy at the time of state switching will be improved, and a predictor that can be used in practical use will be developed.

\section{CONFLICTS OF INTEREST}

The authors declare they have no conflicts of interest.

\section{REFERENCES}

[1] Cabinet Office, Government of Japan, White paper on traffic safety in Japan, 2006, p. 11. Available from: http://www8.cao. go.jp/koutu/taisaku/h18kou_haku/index_pdf.html.

[2] T. Kurinobu, E.T. Harada, T. Daimon, A. Tomito, S. Iwashita, T. Nomura, et al., The effects of electric walking assistance to a walking cart on safety feeling of older adults, Proceedings of the Japanese Society for Cognitive Psychology, Nishi-ku, Fukuoka, Japan, 2015, p. 91 (in Japanese).

[3] RT-Works Corporation. Available from: http://www.rtworks.co.jp/.

[4] T. Yokotani, N. Sato, T. Shimizu, T. Kato, Technique of visualizing walking motion: identification of a part enabling discrimination of motion of a walking assist device user, Proceedings of the Japan Society for System Control Information Conference, Sakyo-ku, Kyoto, Japan, 2018, pp. 263-265 (in Japanese).

\section{Prof. Yoshifumi Morita}

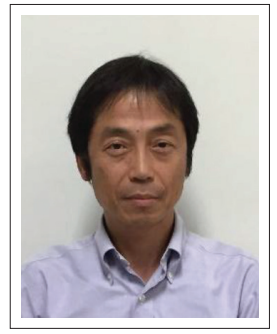

He is a professor in the Department of Electrical and Mechanical Engineering at Nagoya Institute of Technology. He received the $\mathrm{PhD}$ degree from Nagoya Institute of Technology in 1998. His current research interests are rehabilitation support robot/device and robot teaching. 